\title{
SERIES INTRODUCTION \\ The molecular and physiological basis of insulin resistance: emerging implications for metabolic and cardiovascular diseases
}

\author{
Alan R. Saltiel \\ Department of Physiology, University of Michigan School of Medicine, Ann Arbor, Michigan, USA \\ Department of Cell Biology, Parke-Davis Pharmaceutical Research Division, Warner-Lambert Co., Ann Arbor, Michigan, USA \\ Address correspondence to: Alan R. Saltiel, Department of Cell Biology, Parke-Davis Pharmaceutical Research, \\ 2800 Plymouth Road, Ann Arbor, Michigan 48105-2430, USA. Phone: (734) 622-7316; Fax: (734) 622-5668; E-mail: alan.saltiel@wl.com.
}

Following the discovery of insulin by Banting and Best in 1922, it was widely assumed that human diabetes was due exclusively to a deficiency in the secretion of the hormone. However, 10 years later Himsworth (1) noted variations in the responses of diabetic patients to insulin and proposed the notion that insulin insensitivity, not insulin deficiency, was the defining biochemical defect in many diabetics. This idea was not considered seriously until the development of the radioimmunoassay by Berson and Yalow (2), who showed that subjects with adult onset (type 2) diabetes tended to exhibit higher than average levels of circulating insulin. Later studies from Roth (3), Reaven (4, 5), Olefsky (6), and others corroborated these findings and provided a mechanistic basis for the idea that insulin resistance plays a major role in diabetes.

We now know that the pathophysiology of type 2 diabetes involves defects in three organ systems that conspire together to produce abnormal glucose and lipid metabolism (7). While there is some uncertainty regarding the primary lesion and the relative importance of the different tissues, metabolic defects in liver and in peripheral target tissues, such as fat and muscle and pancreatic $\beta$ cells, all contribute to the syndrome. Insulin resistance, which is defined as a state of reduced responsiveness to normal circulating concentrations of insulin, is now recognized as a characteristic trait of type 2 diabetes and contributes to abnormalities in all of these tissues.

Various prospective epidemiological studies across several population groups indicate that type 2 diabetes progresses over a continuum of worsening insulin action, beginning with peripheral insulin resistance and ending with a loss of insulin secretion (Figure 1). In most patients, insulin resistance can be detected long before the deterioration of glucose intolerance occurs. Insulin resistance is a quite common state, associated with aging and a sedentary lifestyle, as well as a genetic predisposition. The state seems to be fueled by, or perhaps to a certain extent the result of, obesity.
The ensuing dysregulation of carbohydrate and lipid metabolism that occurs as a consequence of insulin resistance further exacerbates its progression. $\beta$ cells of the pancreas normally compensate for the insulinresistant state by increasing basal and postprandial insulin secretion. At some point, the $\beta$ cells can no longer compensate, failing to respond appropriately to glucose. This ultimately leads to the deterioration of glucose homeostasis and the development of glucose intolerance. Approximately 5 to $10 \%$ of glucose-intolerant patients in a given year progress to frank diabetes, which continues to worsen as insulin resistance increases. Adipose cells generate more fatty acids, the liver produces more glucose in an unregulated fashion, and the $\beta$ cells undergo complete failure, resulting in the late stages of the disease, where high doses of exogenous insulin may be required.

Even in the absence of diabetes, insulin resistance is a key feature of other human disease states. Impaired insulin action coupled with hyperinsulinemia leads to a variety of abnormalities, including elevated triglycerides, low levels of HDL, enhanced secretion of VLDL, disorders of coagulation, increased vascular resistance,

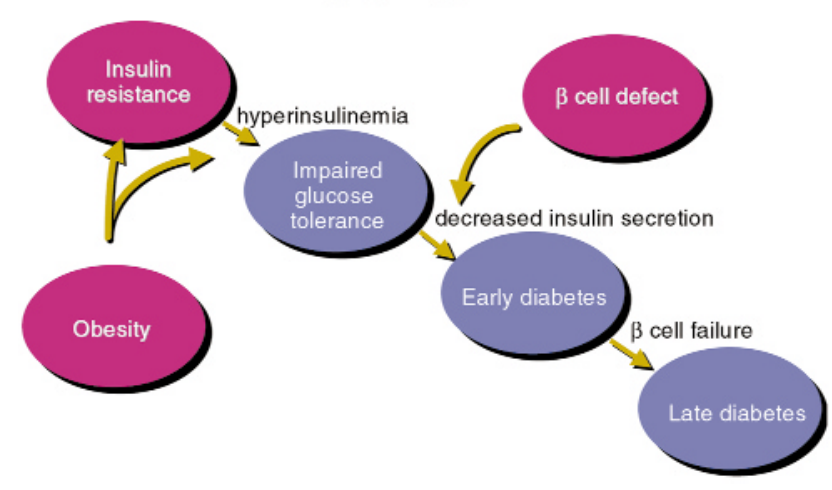

Figure 1

Metabolic staging of Type 2 diabetes 
changes in steroid hormone levels, attenuation of peripheral blood flow, and weight gain. Thus, insulin resistance is often associated with central obesity, hypertension, polycystic ovarian syndrome, dyslipidemia, and atherosclerosis. This constellation of symptoms is often referred to as syndrome $\mathrm{X}$, or insulin resistance syndrome (8). Whether impaired insulin action is directly responsible for all of the symptoms in these patients remains unclear. However, the broad prevalence of insulin resistance and its association with profound metabolic abnormalities are widely accepted.

In this Perspective series, we will attempt to provide some insight into the pathophysiology of insulin resistance. Before considering the molecular basis for this disorder, it is essential to understand the pathways involved in normal cells. Jeffrey Pessin and I discuss the cell biology of insulin action, speculating about possible molecular defects responsible for the attenuation of the actions of the hormone. Gerald Shulman focuses on changes in glucose metabolism that are characteristic of patients and animal models with insulin resistance, in an attempt to identify key alterations in metabolic pathways caused by impaired insulin action. Because it is critical to fully understand the etiology of insulin resistance, Michael Stern hypothesizes about the genetics underlying susceptibility to this state, and the interaction between genes and the environment. Takashi Kadowaki presents an update on animal models of insulin resistance, which have led to startling insights into the basis of this disorder. Kenneth Polonsky and colleagues discuss the interaction between insulin resistance and insulin secretion, exploring the mechanisms leading to states of decompensation that result in diabetes. Jeffrey Flier and Barbara Kahn discuss the complex role of obesity in relation to insulin resistance, concentrating on how the central control of energy uptake and expenditure might also directly influence insulin action in peripheral tissues. The impact of insulin resistance on the cardiovascular system is a critical area for understanding the risk factors associated with this state. Henry Ginsberg surveys this field by escaping from the glucocentric view of most diabetologists, to focus on the regulation of lipid and lipoprotein metabolism. Finally, Jerrold Olefsky discusses new pharmacological approaches designed to enhance insulin action and thus to reverse insulin resistance and the mechanisms by which certain of these drugs may exert their effects.

1. Himsworth, H.P. 1936. Diabetes mellitus: its differentiation into insulinsensitive and insulin insensitive types. Lancet. 1:117-121.

2. Yalow, R.S., and Berson, S.A. 1960. Plasma insulin concentrations in nondiabetic and early diabetic subjects. Diabetes. 4:254-260.

3. Kahn, C.R., et al. 1976. The syndromes of insulin resistance and acanthosis nigricans. Insulin-receptor disorders in man. N. Engl. J. Med. 294:739-745.

4. Olefsky, J., Farquhar, J.W., and Reaven, G. 1973. Relationship between fasting plasma insulin level and resistance to insulin-mediated glucose uptake in normal and diabetic subjects. Diabetes. 22:507-513.

5. Reaven, G.M. 1988. Role of insulin resistance in human disease. Diabetes. 37:1595-1607.

6. Kolterman, O.G., Insel, J., Saekow, M., and Olefsky, J.M. 1980. Mechanisms of insulin resistance in human obesity: evidence for receptor and postreceptor defects. J. Clin. Invest. 65:1272-1284.

7. DeFronzo, R.A. 1987. The triumvirate: B cell, muscle, liver. A collusion responsible for NIDDM. Diabetes. 37:667-687.

8. Reaven, G.M. 1993. Role of insulin resistance in human disease (syndrome X): an expanded definition. Annu. Rev. Med. 44:121-131. 